\title{
Effect of ageing on the morphology and creep and recovery of polymer-modified bitumens
}

\author{
Giacomo Cuciniello • Pietro Leandri · Sara Filippi • Davide Lo Presti • \\ Massimo Losa • Gordon Airey
}

Received: 23 July 2018/Accepted: 25 September 2018/Published online: 3 October 2018

(C) The Author(s) 2018

\begin{abstract}
Polymer additives are used to improve the properties of road bitumens including their oxidative resistance. However, their usage as anti-oxidative materials remains relatively unclear. This study aims to investigate the changes in the morphology and the rheological response of polymer modified bitumens used in road pavement construction caused by ageing. An elastomer (radial styrene butadiene styrene, SBS) and a plastomer (ethyl vinyl acetate, EVA) polymer were mixed with one base bitumen at three polymer concentrations. The bitumens were RTFO and PAV aged. The morphology of the bitumens was captured by fluorescence microscopy while the rheological properties were measured by means of the multiple stress creep and recovery (MSCR) test. The results show that the morphology of the SBS modified bitumen degrades with ageing as a function of polymer concentration and dispersion, with higher dispersion being more resistant. The morphology of the EVA modified bitumen has a low ageing susceptibility irrespective of polymer concentration. The MSCR
\end{abstract}

G. Cuciniello $(\bowtie)$ · D. Lo Presti · G. Airey

Nottingham Transportation Engineering Centre (NTEC), University of Nottingham, Nottingham University Park,

Nottingham NG7 2RD, UK

e-mail: evxgc3@nottingham.ac.uk

P. Leandri · S. Filippi - M. Losa

Department of Civil and Industrial Engineering, University of Pisa, Largo Lucio Lazzarino, 56122 Pisa, PI, Italy response of EVA modified bitumens does not differ from that found for unmodified bitumen, where the hardening produces a decrease in the non-recoverable compliance. In the case of SBS modified bitumen, the degradation of the polymer backbone affects the bitumen hardening as much as the polymer phase dispersed and networked in the bitumen phase. Furthermore, in the case of the elastomer, the average percent recovery is in agreement with the variation of the morphology with ageing. Therefore, the use of the average percent recovery as a valuable rheological index of the integrity of the polymer network can be advocated.

Keywords Polymer-modified bitumen · Ageing · Morphology $\cdot$ Percent recovery $\cdot$ MSCR

\section{Introduction}

In the past few decades, the demand for transportation has increased significantly and road infrastructure is currently challenged by increased traffic. In order to address such challenges, several technologies have been developed to improve the performance of the materials used in road applications. For instance, the modification of bituminous binders with polymeric additives is not recent but represents one of the most successful technologies developed to enhance the performances of asphalt mixtures [1-3]. 
The quality of the modification represents the capability of the polymer to improve the performance of the bitumen when added in small quantities. Specifically, polymer modification aims to stiffen the bitumen and to increase its elasticity at high pavement temperatures; to decrease the temperature susceptibility; to maintain sufficient levels of workability during mix production and laying, and to provide lower levels of stiffness at intermediate and low pavement temperatures. In addition to these modifications, polymermodified bitumens (PMBs) should be stable at storage and ageing resistant [4]. However, such properties need to be considered as the macroscopic result of complex thermodynamic and chemical interactions occurring between the bitumen and the polymer. In particular, the success of the modification depends on the capability of the polymer swelling in the bituminous phase by absorbing the bituminous fractions and thereby having higher compatibility, and in the meanwhile, maintaining its own structure. Such conditions occur with an adequate degree of swelling and solubility of the polymer within the bitumen phase [5]. In this case, the polymer is capable of forming a stable networked structure able to enhance the response of the bitumen. Concerning the affinity, the lighter components of the bitumen appear to be more compatible than the heavier molecules.

Several polymeric additives are available on the worldwide market and they can be classified according to their chemical composition or the target bitumen property. However, two types of polymer are mainly used in bitumen applications: the elastomers that resist deformation by stretching and recovering their shape, and the plastomers that resist deformation by forming a 3-D rigid network. Among the elastomers, the styrene-butadiene-styrene (SBS) block copolymer appears to be the most widely used followed by reclaimed tire. On the other side, among the plastomers, ethyl vinyl acetate (EVA) and Polyethylene are used [6, 7].

Although the response of polymer-modified bitumens has been investigated worldwide [1, 5, 8-16], there are still open questions, drawbacks and concerns. In particular, besides the limitations related to cost and the storage stability, there are concerns about the applicability of the linear viscoelastic (LVE) characterization for the development of dedicated specifications to assess fatigue cracking and rutting resistance $[2,17]$. The Superpave binder specifications
(AASHTO MP1) were developed on unmodified bitumen considering that the pavements would have been designed to maintain the strain levels within the linear regime. Furthermore, several characteristics of polymer-modified bitumen were neglected $[2,4,8,17,18]$. One of the main concerns is that the low strain levels considered in the LVE characterization do not consider the stress-strain dependency of the response of PMBs, and it may provide a poor correlation with mixtures performances. The strain dependency of polymers is given by the fact that when their chains are extended, glassy or crystalline regions and chain entanglement can produce the distortion of the morphology with a consequent change in the physical response [17]. Furthermore, results from mixtures testing, digital image processing, and finite element modelling, have shown that the strain level in the bitumen phase could approach very high strain levels even at low strain levels in the mixtures. Such a difference in the strain level between the mixture and the bitumen phase seems to be caused by a large difference in stiffness between bitumen and aggregates, by localized rotations of aggregates, and by the non-homogeneous distribution of the bitumen film coating the aggregates [8, 19-21].

Besides the enhancement of the mechanical response, part of these additives, including the elastomers, are used by road agencies as anti-oxidative products [2]. However, the effect of polymers on the oxidative mechanisms occurring in PMBs remains relatively unclear and can be considered to be polymer-dependent.

The study of ageing in bitumen encompasses primarily two approaches: the investigation of the chemical changing in the bitumen composition caused by oxidation (microscopic level), and the consequent variations in the mechanical response (macroscopic level). The evaluation of the compositional changes includes different indicators such as the formation of carbonyl compounds measured through fourier infrared spectroscopy (FTIR) [7, 22-28], the variation of the molecular weight distribution of both the polymer and the bitumen phases measured through gel permeation chromatography (GPC) [25, 29], and the formation of asphaltenes [30-32]. Additionally, alternative approaches such as the use of fluorescence microscopy $[33,34]$ and the more recent atomic force microscope [35], provide respectively information on the changes in the morphology and in the microstructure of the 
bitumen. On the other hand, the effect of ageing on the mechanical response is based on ageing indexes that can be measured through empirical parameters (e.g., softening point) and rheological parameters (e.g., viscosity, complex modulus, phase angle) at different ageing conditions [36-39].

In the case of unmodified bitumen, the mechanism of oxidation appears to be relatively clear. At the microscopic level, it can be seen as the diffusion of oxygen through a thin film of bitumen producing the formation of oxygen-containing functional groups (i.e., carbonyl compounds) with strong interactive forces. The presence of these forces drives the formation of heavy molecular compounds, polar in nature and characterized by stronger molecular interactions that cause a reduction in the molecular mobility under shear stress [40-45]. At the macroscopic level, such a reduced molecular mobility reduces the strain tolerance of the bitumen, increasing the risk of premature fatigue and/or low-temperature cracking.

In the case of PMBs, although the study of the effect of polymers on bitumen oxidation is not recent [46], the complexity of the interactions between polymer and bitumen does not allow a relatively well defined conclusion [7, 22-39, 47-52]. Results from compositional analysis and dynamic mechanical analysis (DMA) show that for PMBs the definition of a unique ageing mechanism could be inaccurate since the effect of the oxidative processes is polymer-dependent. Nevertheless, general assessments for some polymers can be provided. For instance, it appears that the presence of the SBS reduces in some cases the formation of carbonyl compounds and sulfoxide with the consequent reduction in the formation of strong interactive polar functional groups [53]. Besides this, the analysis of the molecular weight distribution shows that the SBS polymer undergoes degradation due to thermo-oxidative processes. Such changes are reflected in the rheological response of the PMB where the deterioration of the polymer induces a more viscous response that might mitigate the hardening of the bitumen phase.

However, as per the considerations included above, the linear viscoelastic characterization of modified bitumen might not be indicative of the effective contribution of the polymer on the PMB response since at low strain levels, the polymer chains do not show any slippage or change in morphology. In fact, although the degradation of the polymer backbone could mitigate the hardening of the bitumen in terms of complex modulus and viscosity, the effect of such degradation on the expected polymer contribution measured at higher strain levels and under a higher number of loading cycles remains unclear [50-52].

Therefore, the assessment of the durability of PMBs should be conducted considering the simultaneous effect of strain levels (high enough to cause slippage in the polymer chains) and ageing.

The aim of this paper is to investigate the ageing in PMBs, trying to identify what changes occur in the polymer network and which are the effects on the rheological response measured at high strain levels. To achieve this aim, images from fluorescence microscopy are used to provide rational indicators on how the polymer network changes with ageing. Specifically, the analysis of the morphology aims to provide a qualitative assessment of the effect of ageing on the polymer dispersed in the bituminous phase.

In order to investigate the effects of ageing on the mechanical response of PMBs measured at strain levels higher than those considered in the LVE characterization, the multiple stress creep and recovery (MSCR) test, conducted as per the AASHTO T-350, has been used. In particular, this test aims to identify the effect of laboratory simulated ageing processes on the change in the non-recoverable compliance $\left(J_{\mathrm{nr}}\right)$ and on the average percent recovery $(R)$, both measured at a stress level of $3.2 \mathrm{kPa}$. Although the magnitude of this stress level may not guarantee response in the non-linear region in some cases, in this study it has been selected for two specific reasons. The first is due to the fact that $3.2 \mathrm{kPa}$ is used as the reference stress level in the current specifications of the MSCR test (AASHTO M332). The second is because at such stress levels many PMBs show slippage of polymer chains in the test [17]. Although the relations between bitumen elasticity and mixture performance are not fully understood, the use of average percent recovery $(\% R)$ as a quality indicator of the polymer efficiency and mixture durability can be advocated [54, 55]. The elasticity of rubber can be defined as the capacity of complete large deformation recover. This property is a function of molecular requirements such as the flexibility of polymer chains and the presence of a structured network. In the case of PMBs, the formation of this network depends on several factors such as the polymer concentration, the 
blending process, the use of stabilizing cross-linkers, and the affinity between polymer and bitumen. Therefore, the capacity of this network to resist the effect of thermo-oxidative ageing processes could be evaluated through its capacity of maintaining the strain recovery at different levels of ageing.

\section{Experimental methodology}

\subsection{Test methods}

\subsubsection{Laboratory simulated ageing}

The bitumens were laboratory short-term aged in the rolling thin film oven (RTFO) and long-term aged in the pressure ageing vessel (PAV) by following the AASHTO R28. Besides the PAV exposure of $20 \mathrm{~h}$ (1 PAV) prescribed by the standard, different levels of long-term ageing were simulated by using different times of PAV exposure (i.e. $10 \mathrm{~h}$ referred to as 0.5 $\mathrm{PAV}$ and $40 \mathrm{~h}$ referred to as $2 \mathrm{PAV}$ ) at $100{ }^{\circ} \mathrm{C}$.

\subsubsection{Fluorescence microscopy}

A Leica ${ }^{\circledR}$ DM LB fluorescence microscope was used to capture images of the PMB morphology at $10 \times$ magnification. Although the morphology provides a qualitative description of the polymer dispersion within the bituminous phase, its analysis provides information on the affinity between the polymer and the bitumen. Furthermore, the changing in the polymer dispersion with ageing can provide a qualitative description of the degradation of the polymer network to justify the variation in the percent recovery and $J_{\mathrm{nr}}$ measured in the MSCR.

In the sample preparation, stripes of bitumen of about $10 \mathrm{~mm} \times 50 \mathrm{~mm} \times 2 \mathrm{~mm}$ are refrigerated for $10 \mathrm{~h}$ to freeze the morphology. After that, the stripes are cut (brittle cracked) in the middle so as not to affect the morphology. The surface corresponding to the crack is attached to the glass used in the microscope. To obtain representative images, 9 surfaces per bitumen were prepared for observation under the microscope.

\subsubsection{Multiple stress creep and recovery (MSCR)}

The MSCR was conducted as per AASHTO T-350. The test was conducted at two stress levels: $0.1 \mathrm{kPa}$ and $3.2 \mathrm{kPa}$. At each stress level, 10 creep and recovery cycles were applied. The values of $J_{\mathrm{nr}}$ and $R$ were calculated at each loading cycle and then averaged at each stress level.

The non-recoverable compliance $\left(J_{\mathrm{nr}}\right)$ at the $n$ cycle was calculated using Eq. (1).

$J_{\mathrm{nr}}^{n}\left(\mathrm{kPa}^{-1}\right)=\frac{\varepsilon_{\mathrm{r}}^{n}-\varepsilon_{0}^{n}}{\tau_{0}}$

where $\varepsilon_{\mathrm{r}}^{n}$ is the strain value at the end of the recovery phase; $\varepsilon_{0}^{n}$ is the initial strain value at the beginning of the creep portion; $\tau_{0}$ is the value of the stress level used in the loading cycle.

The percent recovery $(R)$ at the $n$-cycle was calculated using Eq. (2).

$R^{n}(\%)=\frac{\varepsilon_{\mathrm{c}}^{n}-\varepsilon_{\mathrm{r}}^{n}}{\varepsilon_{\mathrm{c}}^{n}-\varepsilon_{0}^{n}}$

where $\varepsilon_{\mathrm{c}}^{n}$ is the strain value at the end of the creep portion.

According to AASHTO M 332, the traffic designation is determined as a function of average $J_{\mathrm{nr}}$ value calculated at the stress level of $3.2 \mathrm{kPa}$. Therefore, the results incorporated in this paper refer to the stress level of $3.2 \mathrm{kPa}$.

The $J_{\mathrm{nr}}$ (measured at $3.2 \mathrm{kPa}$ ) is considered as an indicator of the susceptibility of the bitumen to the accumulation of permanent deformation measured at higher strain levels than those used in the LVE analysis. It is known that MSCR is conducted to assess the bitumen's potential for accumulation of permanent deformation that typically occurs at an early stage in the pavement life. Therefore, the use of this test on long-term aged bitumen might not be representative of pavement field conditions. However, according to AASHTO M 332, the MSCR test is conducted on the RTFO residue only. As a consequence, the traffic designations (i.e., Standard-S, Hard-H, Extreme$\mathrm{E}$ and Very Extreme-V) are measured on a single level of ageing and the combined effects of polymer degradation and bitumen hardening using this classification are not documented. The MSCR test was conducted as per the AASHTO specifications for the sake of consistency with the standards used for the 
PAV ageing (AASHTO R 28) and PG-grading (AASHTO M 320).

The testing temperatures (given in Fig. 3) were selected as the temperature at which the parameter $G^{*}$ / $\sin \delta$ measured on the RTFO residue is equal to $2.20 \mathrm{kPa}$ (defined as Continuous Grade High Temperature). This criterion was selected to compare the different bitumen at relatively similar levels of stiffness after short-term ageing. It is worth mentioning that according to AASHTO M 332, the traffic designation should be measured at the Performance Grade temperature and not at the Continuous Grade High Temperature. However, the traffic designation is included to provide a synthetic indicator of the change in the resistance to the accumulation of permanent deformation and not for grading purposes.

\section{Materials}

Six PMBs were produced by mixing the control bitumen Penetration grade (Pen) 70-100 with two types of polymer: an elastomer (SBS-polystyrene content $29-31 \%$ ) and a plastomer (EVA-28\% by weight vinyl acetate). For each polymer, three concentrations by weight were used: $2 \%$ (medium-low content), 4\% (high content) and 6\% (very high content). In the case of modification with SBS, organic sulphur was used in a concentration of $0.1 \%$ with respect to the polymer + bitumen weight as a stabilizer. The high-temperature continuous grade (HTPG) of each bitumen was measured as per the AASHTO M320 Designation.
The job-mix formula and the high-temperature continuous grades are given in Table 1 .

As shown by the continuous grade temperatures, the SBS seems to be more effective than the EVA in providing the high-temperature bump grade. This is not surprising since the SBS works as an elastomer capable of providing adequate levels of stiffness and elasticity at high temperatures. On the other hand, the EVA is a plastomer that provides stiffening effects in the range of temperatures below its melting point without necessarily providing any further elastic recovery. The melting point of the EVA used in this study is $74{ }^{\circ} \mathrm{C}$. As shown by the results in Table 1 , since the grading temperatures are close to the melting point of the polymer, the stiffening effect of the EVA is limited irrespective of the polymer concentration. However, this aspect is not of concern since this work aims to compare the effect of ageing on different polymers at the same concentration but not the same High-Temperature PG grade.

\section{Results and discussion}

This section includes the description of the morphology of the bitumens at different levels of ageing and the results of the MSCR test.

\subsection{Fluorescence microscopy}

The morphologies of the PMB after modification are considered as a reference in their unaged condition and are shown in Fig. 1.

Table 1 Bitumen modification methods

\begin{tabular}{|c|c|c|c|c|c|c|}
\hline Bitumen & $\begin{array}{l}\text { Base } \\
\text { bitumen }\end{array}$ & $\begin{array}{l}\text { Polymer concentration } \\
(\%)\end{array}$ & $\begin{array}{l}\text { Chemical } \\
\text { stabilizer }\end{array}$ & $\begin{array}{l}\text { Mixing temperature } \\
\left({ }^{\circ} \mathrm{C}\right)\end{array}$ & $\begin{array}{l}\text { Mixing time } \\
(\min )\end{array}$ & $\begin{array}{l}\text { HT-PG } \\
\left({ }^{\circ} \mathrm{C}\right)\end{array}$ \\
\hline $\begin{array}{l}\text { Pen } \\
\quad 70-100\end{array}$ & - & - & - & - & - & 64.3 \\
\hline SBS 2 & Pen $70-100$ & 2 & $0.1 \% \mathrm{~S}$ & 180 & 180 & 69.2 \\
\hline SBS 4 & & 4 & $0.1 \% \mathrm{~S}$ & 180 & 180 & 74.8 \\
\hline SBS 6 & & 6 & $0.1 \% \mathrm{~S}$ & 180 & 180 & 86.7 \\
\hline EVA 2 & & 2 & - & 180 & 120 & 66.2 \\
\hline EVA 4 & & 4 & - & 180 & 120 & 69.6 \\
\hline EVA 6 & & 6 & - & 180 & 120 & 70.5 \\
\hline
\end{tabular}




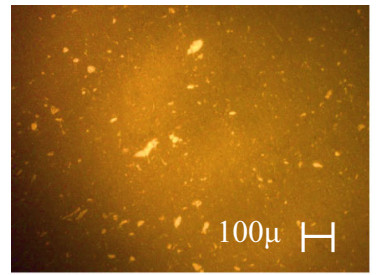

(a) SBS 2

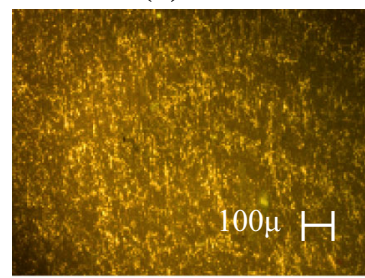

(c) SBS 4

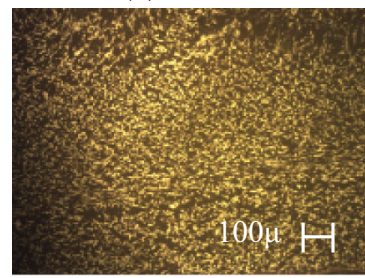

(e) SBS 6

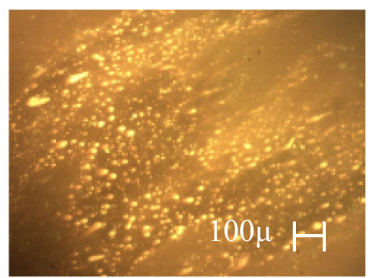

(b) EVA 2

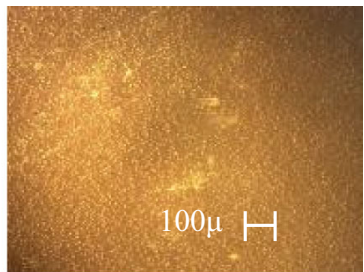

(d) - EVA 4

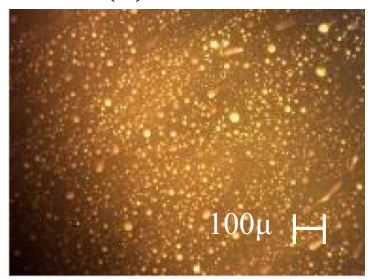

(f) EVA 6
Fig. 1 Morphology of polymer modified bitumen after modification $(10 \times$ magnification $)$

The SBS and EVA polymers show different morphologies. As shown in Fig. 1b, d, f, the EVA remains dispersed within the bituminous phase as disconnected spherical droplets irrespective of the polymer concentration. However, at 2\% EVA (Fig. 1b) such droplets do not have the same dispersion as the other two levels. The spherical shape of the droplets indicates somehow a low compatibility with the base bitumen since the sphere is the shape with the lowest specific surface. In other words, this shape minimizes the interface, and thus the interactions, between the two phases. In light of this, the polymer and the bitumen phases, although coexisting, remain separated without forming any network. In addition, the spherical shape of the polymer might reduce the surface of the polymer exposed to oxygen diffusion during oxidation. However, the lack of formation of a true polymer network correlates with the mechanical response of EVA modified bitumen in which the polymer behaves as a reinforcement only without necessarily increasing the elasticity of the bitumen.

The morphology of the SBS modified bitumens (Fig. 1a, c, e) depends on the polymer concentration. At $2 \%$ SBS (Fig. 1a), the polymer is not capable of forming a visible interconnected network and the particles remain dispersed within the bituminous phase. At $4 \%$ and $6 \%$ SBS (Fig. 1b, c), the polymer forms a structured network throughout the bituminous phase becoming the dominant phase. The formation of this network is clearer at 6\% SBS (Fig. 1c). This network is constituted of elongated interconnected branches, revealing an adequate level of affinity with the base bitumen, but, on the other hand, the wider polymer surface is exposed to oxygen diffusion. Aside from the polymer concentration, the formation of the polymer network is enhanced by the use of sulphur as a cross-linker.

The figures below show the morphology of the SBS modified bitumens after laboratory simulated ageing (Fig. 2). The RTFO-aged (Fig. 2a-c) condition is representative of the morphology after short-term ageing, whereas the PAV aged conditions (Fig. 2d-f) are representative of the residual morphology at a high level of oxidation.

The effect of ageing on the morphology of the SBS modified bitumen depends on the concentration. At $2 \%$ SBS the polymer phase seems to completely degrade after RTFO ageing (Fig. 2a). Similarly, the

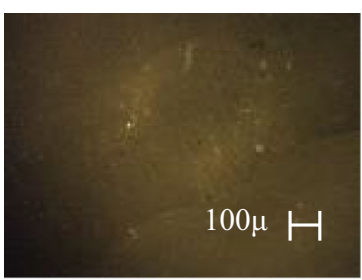

(a) SBS 2 - RTFO

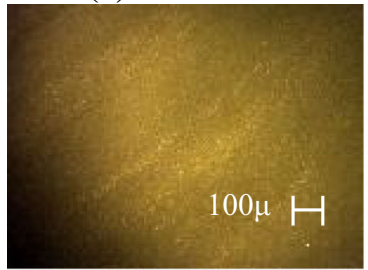

(b) SBS 4 - RTFO

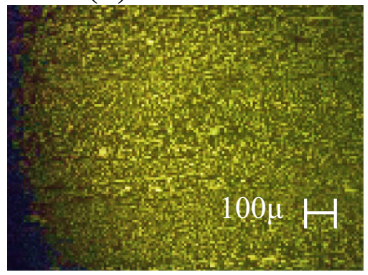

(c) SBS 6 - RTFO

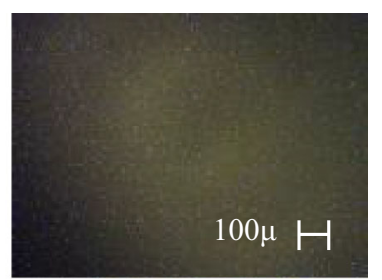

(d) SBS 2 -PAV

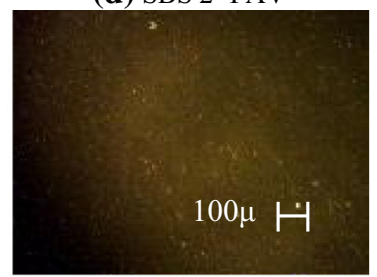

(e) SBS 4 - PAV

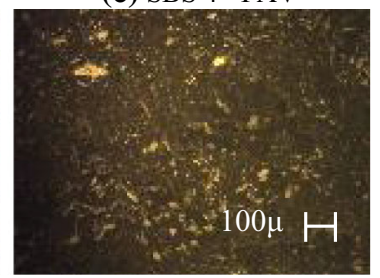

(f) SBS 6 - PAV
Fig. 2 Residual morphology of SBS modified bitumen at different levels of ageing $(10 \times$ magnification $)$ 
morphology of the 4\% SBS bitumen after RTFO (Fig. 2b) is different from the morphology shown after modification (Fig. 1c). After RTFO, the branched structure dissolves in a dispersion of thin particles (Fig. 2b). The polymer phase then disappears after a single PAV cycle (Fig. 2e).

In the case of the $6 \%$ SBS bitumen, the morphology after RTFO (Fig. 2c) does not show any relevant variation compared to the unaged conditions (Fig. 1f). However, this aspect does not necessarily mean that at high polymer concentration, the polymer network is not sensitive to short-term ageing, but could be linked to the effect of the bitumen viscosity during the ageing tests. The viscosity of the $6 \%$ SBS modified bitumen RTFO-aged at $163{ }^{\circ} \mathrm{C}$ (RTFO temperature) was $1.4 \mathrm{~Pa}$ s. This value could be high enough to cause the bitumen to form a thin film in the RTFO bottle with a consequent reduction of the RTFO effects. Nonetheless, as per the images shown in Fig. $2 f$, it is clear that the oxidative processes in the PAV cause the degradation of the SBS network. It is well known that SBS shows a reactivity with oxygen due to the presence of unsaturated $\mathrm{C}=\mathrm{C}$ double bonds in the Polybutadiene blocks [25, 26, 28]. Although the polymer degrades with oxidation, the polymer phase is still visible in the morphology after 1 cycle of PAV ageing (Fig. 2f). However, at $6 \%$ SBS concentration, the effect of the PAV ageing might be mitigated by the "viscosity effect" at the PAV temperature, typical of polymermodified binders [56].

The morphology of the EVA modified bitumens does not seem to be significantly affected by both the RTFO and the PAV ageing. At $2 \%$ polymer concentration, the polymer particles remain dispersed as small spherical droplets distributed within the bituminous phase even at high levels of ageing. Such morphology does not seem to differ significantly from the unaged conditions (Fig. 1b). Also at $4 \%$ and $6 \%$ polymer concentration, the morphology constituted of sparsely dispersed particles does not vary with ageing remaining similar to the morphologies observed in Fig. 1d, e. The lower sensitivity of this polymer to oxidation compared to the SBS, is due to the absence of $\mathrm{C}=\mathrm{C}$ bonds in the chain which makes the EVA less prone to react with the oxygen molecules.

\subsection{MSCR — analysis of $J_{\mathrm{nr}}$ values}

The values of the $J_{\mathrm{nr}}$ at the stress level of $3.2 \mathrm{kPa}$ measured at the high continuous grade temperature are given in Fig. 3.

The non-recoverable compliance of the Pen bitumen decreases with ageing. This result is not surprising since the stiffening effect of ageing on unmodified bitumen is well known. The variation of the $J_{\mathrm{nr}}$ value with the ageing of the SBS modified bitumens depends clearly on the polymer concentration. In the case of SBS 2, the $J_{\mathrm{nr}}$ decreases with ageing as seen as for the Pen bitumen. The traffic designation varies from an $\mathrm{S}$-grade at the unaged condition, to a $\mathrm{V}$-grade after 2 cycles of PAV. At $4 \%$ SBS concentration, the $J_{\mathrm{nr}}$ increases after RTFO ageing and then it remains relatively constant throughout the oxidative processes in the PAV. This concentration seems to represent a sort of optimum polymer content that minimizes the oxidative susceptibility of the modified bitumen. In fact, the $J_{\mathrm{nr}}$ values at the different PAV exposure times do not show a significant variation, with the traffic designation remaining constant.

The SBS 6 bitumen shows an inverse behaviour compared to the Pen and the SBS 2 bitumens. In this case, the $J_{\mathrm{nr}}$ increases with ageing with the traffic designation varying from a V-grade, at unaged conditions, to an S-grade after 2 cycles of PAV. The majority of the degradation occurs in the PAV, with the RTFO producing a marginal effect only. The behaviour may be related to the high viscosity of the bitumen at the RTFO temperature. Therefore, at the selected testing conditions, the different SBS concentrations produce different variations of $J_{\mathrm{nr}}$ with ageing. Such differences might depend on the level of polymer dispersion in the bituminous phase and on its capability of forming a dominant network. In the case of the medium-low polymer concentration (2\% SBS), as shown in Fig. 1a, the polymer phase is not dominant with respect to the bituminous phase. Therefore, although the polymer undergoes degradation with ageing, the loss of its properties only marginally affects the response of the binder that appears to be governed more by the bituminous phase stiffened by ageing. At higher polymer concentration (4\% SBS), the response of the modified bitumen at the different levels of ageing seems to be determined by both the processes occurring in the biphasic material. Except for an initial worsening of the response after the 


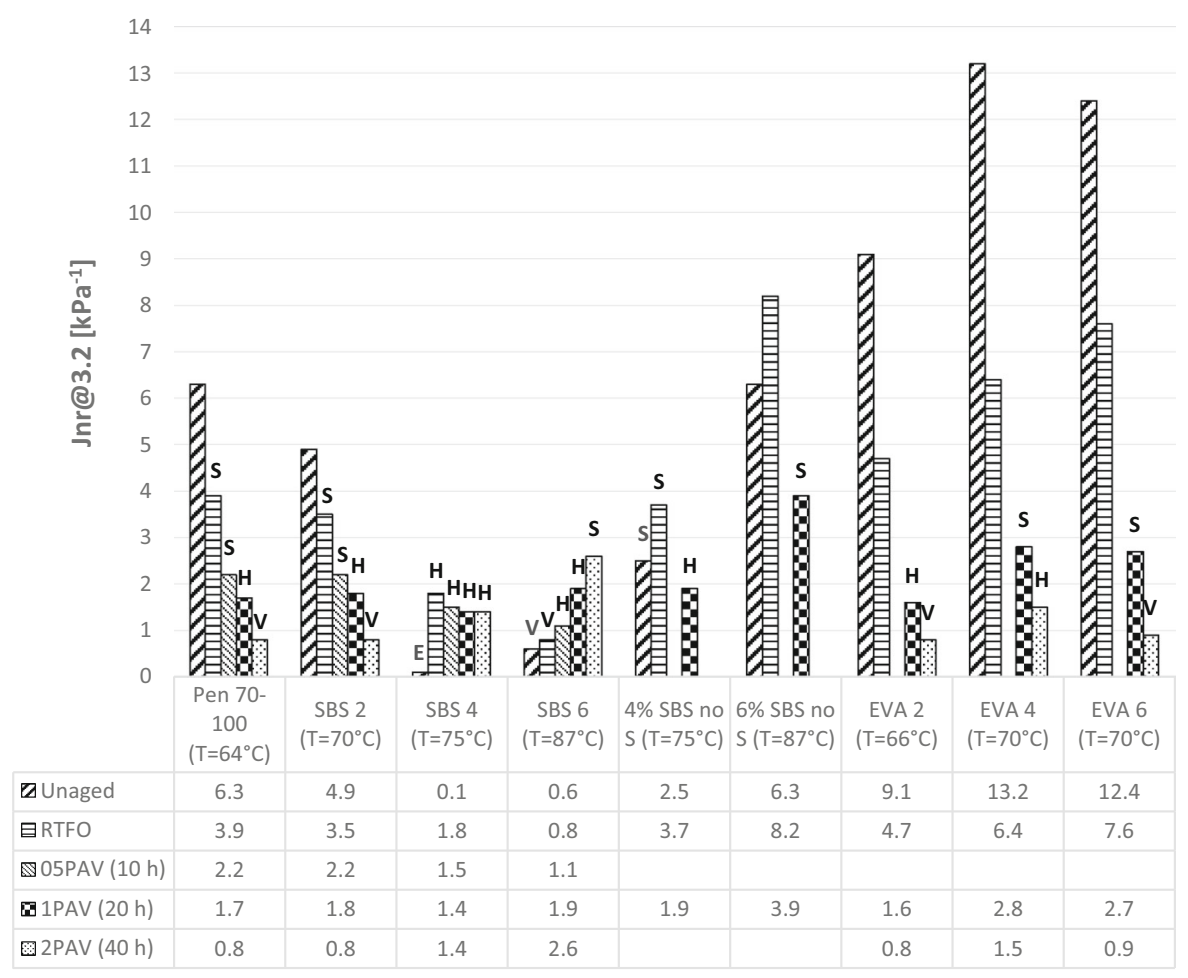

Fig. $3 J_{\mathrm{nr}} @ 3.2 \mathrm{kPa}\left(\mathrm{kPa}^{-1}\right)$ measured at high continuous grade temperature [AASHTO M 332 traffic designation: S-Standard $\left(J_{\text {nr max }}=4.5 \mathrm{kPa}^{-1}\right) ; \mathrm{H}$-Heavy $\left(J_{\mathrm{nr} \max }=2 \mathrm{kPa}^{-1}\right) ; \mathrm{V}-$ Very Heavy $\left.\left(J_{\mathrm{nr} \max }=1 \mathrm{kPa}^{-1}\right) ; \mathrm{E}-\operatorname{Extreme}\left(J_{\mathrm{nr}} \max =0.5 \mathrm{kPa}^{-1}\right)\right]$

RTFO, the degradation of the polymer phase, that is initially well dispersed in the bitumen (Fig. 1c), seems to compensate the stiffening of the bituminous phase maintaining constant $J_{\mathrm{nr}}$ values through ageing. However, asides from the degradation, the polymer might mitigate the stiffening of the bitumen phase by acting as an anti-oxidant and preventing the $J_{\mathrm{nr}}$ from increasing at higher levels. At 6\% SBS concentration and under the selected testing temperatures, the effect of the polymer network (Fig. 1e) on the response of the modified bitumen seems to become dominant compared to the bituminous phase. Although the bitumen phase becomes stiffer with ageing, the degradation of the polymer network provides a more relevant effect on the response under loading causing the increase of the $J_{\text {nr }}$ value.

Based on these results, it seems that the more the polymer network is dispersed in the bitumen phase, the more its degradation can compensate the stiffening of the bitumen phase. It is important to refer to the formation of the polymer network and not to the polymer concentration only. In fact, results of the MSCR test conducted on a $6 \%$ SBS and a $4 \%$ SBS modified bitumen, without the cross-linker, exhibit a different trend of the $J_{\mathrm{nr}}$ value with ageing (Fig. 3). In this case, the MSCR test was conducted at the continuous grade temperatures of the bitumens with the same polymer concentrations modified with Sulphur $\left(6 \% \mathrm{SBS}-87^{\circ} \mathrm{C} ; 4 \% \mathrm{SBS}-75^{\circ} \mathrm{C}\right)$.

On the other hand, the results in Fig. 3 demonstrate that the EVA does not enhance the traffic designation of the binder irrespective of the polymer concentration. Furthermore, the increase in the testing temperature caused by the stiffening effect of the polymer produces a higher $J_{\mathrm{nr}}$ value compared to the unmodified bitumen. However, with this polymer, the variation of the $J_{\mathrm{nr}}$ values at the different levels of ageing does not seem to be affected by the polymer or polymer content with the $J_{\mathrm{nr}}$ values decreasing with ageing. The $J_{\mathrm{nr}}$ values with the ageing of the EVA modified bitumens does not show any deviation from the trend shown by the Pen bitumen. However, the morphologies of EVA remain unvaried from the unaged conditions through to the different levels of ageing. This aspect highlights that the polymer dispersion is not affected by the thermo-oxidative 
processes occurring in the RTFO and in the PAV. Such a reduced degradation indicates a low oxidative susceptibility of the polymer. Nevertheless, although the polymer does not deteriorate, it does not seem to provide any remarkable contribution to mitigating the stiffening effect of ageing on the bituminous phase measured as per the $J_{\mathrm{nr}}$ values. This is not surprising, considering the low interactions evidenced by fluorescence microscopy.

\subsection{MSCR — analysis of average percent recovery $(\% R)$}

Besides the non-recoverable compliance, the average percent recovery $(R)$ has been used to assess the effect of ageing on the elasticity of the polymer network since it represents a qualitative indicator to determine the presence of elastomers (AASHTO TP 70). The variation of percent recovery at the $3.2 \mathrm{kPa}$ stress level of the SBS and EVA modified bitumens are given in Fig. 4.

The Pen bitumen does not show any relevant level of percent recovery even at very high levels of oxidation. On the other hand, the presence of the SBS polymer increases the elasticity of the bitumen as a function of the polymer concentration:

- The $2 \%$ SBS concentration produces a marginal increase in elasticity with respect to the unmodified bitumen irrespective of the level of ageing. In this case, the lack of formation of the polymer network after modification might be the cause of a limited increase in elasticity at the testing temperature.

- The SBS 4 bitumen shows an important increase in elasticity compared to the unmodified bitumen ( $R=95 \%$ at unaged conditions). Besides this, it is interesting to show how the RTFO reduces the $\% R$ value by more than $50 \%$ from its initial value. Such a loss in recovery depends on the degradation of the polymer network shown in Fig. 2b. Afterwards, although the oxidative process occurring in the PAV seems to deteriorate the polymer network (Fig. 2e), the SBS 4 bitumen exhibits a limited reduction in recovery within the 1 st cycle of PAV $(-8 \%)$. This result is consistent with the trend of the $J_{\mathrm{nr}}$ values. Then, after the second cycle of PAV, the polymer network loses its residual elasticity and the value of the percent recovery drops to $18 \%$.

- The SBS 6 bitumen shows, at the unaged condition, a lower value of recovery compared to the SBS 4 . The reason behind this, could be the higher testing temperature selected for this bitumen. In this case, the loss in recovery starts with the oxidative process in the PAV while the RTFO produces a negligible effect only. It is interesting to observe how the polymer morphology correlates

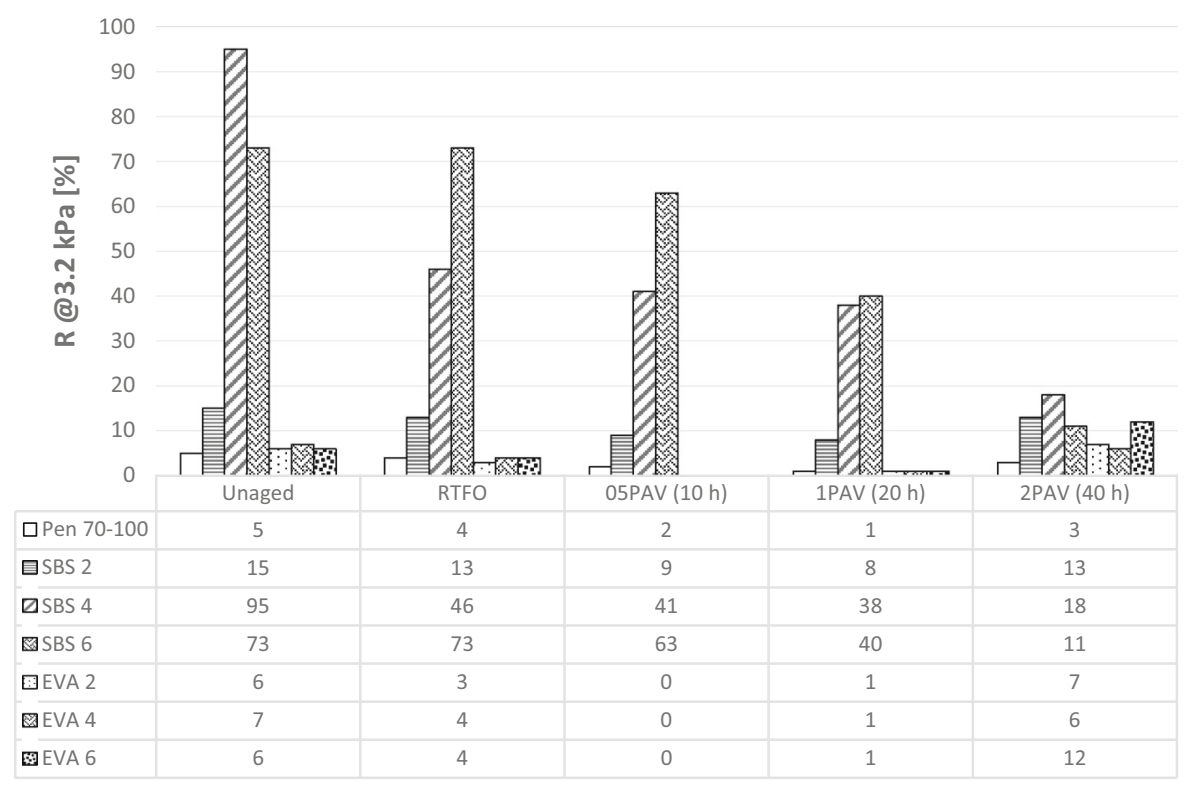

Fig.4\%R@3.2 kPa (\%) measured at high continuous grade temperature 
well with the value of the percent recovery. At the same value of percent recovery, the morphology of the SBS 6 bitumen at the unaged condition (Fig. 1e) is identical to the morphology at the RTFO aged condition (Fig. 2c).

As expected, the EVA polymer does not produce any relevant increase in the bitumen elasticity irrespective of the polymer concentration. However, at very high oxidative levels ( 2 PAV aged bitumen), the percent recovery seems to increase proportionally to the EVA content. Such a gain in recovery, although limited, might be attributed to the EVA concentration at this very high oxidative state. However, such considerations need to be taken at the interpretative level only, since the difference between the percent recovery of the Pen bitumen and the EVA modified bitumens is not remarkable. As seen as for the $J_{\mathrm{nr}}$ values, it can be concluded through the measure of the percent recovery that, although its morphology remains consistent throughout ageing, the EVA polymer does not alter the ageing behaviour of the base bitumen.

\section{Conclusions}

Six polymer-modified bitumens were prepared by mixing a Pen 70-100 base bitumen with three concentrations of two polymers: an elastomer (radial SBS-polystyrene content 29-31\%), and a plastomer (EVA-28\% by weight vinyl acetate). The bitumens were laboratory-aged combining the RTFO and different times of PAV exposure.

The images from the fluorescence microscope were used to support the findings from the MSCR test. The use of fluorescent microscopy provides a qualitative estimation of the degradation of the morphology with ageing. The Multiple Stress Creep and Recovery (MSCR) test was performed to evaluate the effect of the thermo-oxidative degradation of the polymer network on the non-recoverable compliance $\left(J_{\mathrm{nr}}\right)$ and the average percent recovery $(\% R)$ measured at $3.2 \mathrm{kPa}$ at the continuous grade temperature.

The results and the analysis allow the following concluding remarks to be drawn:

- Based on the morphology of the EVA modified bitumens, it seems that polymer does not form a networked structure in the bituminous phase, but it remains dispersed as disconnected spherical droplets irrespective of the polymer concentration. The morphology of the EVA modified bitumens does not show a significant oxidative susceptibility with the polymer dispersion remaining unaltered with ageing irrespective of the polymer concentration.

- The morphology of the SBS polymer network degrades with ageing as a function of polymer concentration and dispersion. The network resistance seems to increase with polymer concentration and dispersion. However, at very high SBS content, the high level of viscosity (and possibly of surface tension) may mitigate the effects of the RTFO and the PAV ageing.

- A comparative analysis of the morphology of the SBS and the EVA modified bitumens used in this study highlights that the two polymers have a different ageing susceptibility with the EVA being more resistant than the SBS. However, the ageing susceptibility of the SBS polymer has been remarked by other authors.

- The viscoelastic parameters selected from the MSCR test are non-recoverable compliance $\left(J_{\mathrm{nr}}\right)$ and the percent recovery $(R)$ measured at $3.2 \mathrm{kPa}$.

- In the case of unmodified bitumen, the stiffening effect of ageing produces a decrease in the $J_{\text {nr }}$ strain without any remarkable effects on elasticity.

- In the EVA modified bitumens, although the polymer dispersion shows a low oxidative susceptibility, the polymer does not affect the reduction in $J_{\mathrm{nr}}$ produced by the stiffening of the base bitumen.

- In case of SBS modified bitumens, the more the polymer phase is dispersed and structured, the more the polymer (and its degradation) can mitigate the stiffening of the bitumen. In fact, at medium-low SBS content (2\%), the lack of formation of a networked structure determines the variation of the $J_{\mathrm{nr}}$ with ageing follows the same trend as the unmodified bitumen. At high SBS content (4\%), the degradation of the polymer compensates the stiffening of the bitumen, maintaining the $J_{\mathrm{nr}}$ value constant with oxidation. At very high SBS content (6\%), the polymer network becomes so dominant that its degradation somehow overcomes the 
bitumen hardening resulting in an increase in the $J_{\mathrm{nr}}$ value.

- As expected, in the case of elastomers, the percent recovery is connected with the integrity of the polymer network observed with the microscope.

\section{Compliance with ethical standards}

Conflict of interest The authors declare that they have no conflict of interest.

Human and animal rights This research does not include any study with humans and animals performed by any of the authors.

Open Access This article is distributed under the terms of the Creative Commons Attribution 4.0 International License (http:// creativecommons.org/licenses/by/4.0/), which permits unrestricted use, distribution, and reproduction in any medium, provided you give appropriate credit to the original author(s) and the source, provide a link to the Creative Commons license, and indicate if changes were made.

\section{References}

1. Yildirim Y (2007) Polymer modified asphalt binders. Constr Build Mater 21(1):66-72

2. Bahia HU, Hanson DI, Zeng M, Zhai H, Khatri MA, Anderson M (2001) National highway research programme (NCHRP) report 459—characterization of modified asphalt binder in Superpave mix design

3. Planche JP (2011) Europe's modified asphalt experiences. In: 12th annual meeting, Association of Modified Asphalt Producers (AMAP), Kansas City

4. Bahia HU, Perdomo D (1996) Current practices for modification of paving asphalts. Fuel 41:4

5. Polacco G, Filippi S, Merusi F, Stastna G (2015) A review of the fundamentals of polymer-modified asphalts: asphalt/ polymer interactions and principles of compatibility. Adv Coll Interface Sci 224:72-112

6. Becker Y, Mendez MP, Rodriguez Y (2001) Polymer modified asphalt. Vis Tecnol 9(1):39-50

7. Lucena M, Soares S, Soares J (2004) Characterization and thermal behavior of polymer-modified asphalt. Mater Res 7(4):529-534

8. Bahia HU, Perdomo D, Turner P (1997) Applicability of Superpave binder testing protocols to modified binders. Transp Res Rec J Transp Res Board 1586:16-23

9. Zhu J, Birgisson B, Kringos N (2014) Polymer modification of bitumen: advances and challenges. Eur Polymer $\mathbf{J}$ 54:18-38

10. Lesueur D (2009) The colloidal structure of bitumen: consequences on the rheology and on the mechanisms of bitumen modification. Adv Coll Interface Sci 145(1-2):42-82
11. Airey GD (2002) Rheological evaluation of ethylene vinyl acetate polymer modified bitumens. Constr Build Mater $16(8): 473-487$

12. Airey GD (2003) Rheological properties of styrene butadiene styrene polymer modified road bitumens. Fuel 82(14):1709-1719

13. Merusi F, Giuliani F, Polacco G (2012) Linear viscoelastic behaviour of asphalt binders modified with polymer/clay nanocomposites. Procedia Soc Behav Sci 53:335-345

14. Polacco G, Kř́ž P, Filippi S, Stastna G, Biondi D, Zanzotto L (2008) Rheological properties of asphalt/SBS/clay blends. Eur Polymer J 44(11):3512-3521

15. Lo Presti D (2013) Recycled tyre rubber modified bitumens for road asphalt mixtures: a literature review. Constr Build Mater 49:863-881

16. Mandal T, Sylla R, Bahia HU, Barmand S (2015) Effect of cross-linking agents on the rheological properties of polymer-modified bitumen. Road Mater Pavement Des 16(1):349-361

17. D'Angelo J, Kluttz R (2007) Revision of the Superpave high temperature binder specification: the multiple stress creep recovery test (with discussion). J Assoc Asphalt Paving Technol 76:123-162

18. Bahia HU, Zhai H, Onnetti K, Kose S Non-linear viscoelastic and fatigue properties of asphalt binders. https:// trid.trb.org/view/639703

19. Kose S, Guler M, Bahia HU, Masad E (2000) Distribution of strains within hot-mix asphalt binders: applying imaging and finite-element techniques. Transp Res Rec J Transp Res Board 1728:21-27

20. Masad E, Somadevan N (2002) Microstructural finite-element analysis of influence of localized strain distribution on asphalt mix properties. J Eng Mech 128(10):1105-1114

21. Airey GD, Rahimzadeh B, Collop A (2004) Linear rheological behavior of bituminous paving materials. J Mater Civ Eng 16(3):212-220

22. Tabatabaei SA (2013) Evaluate aging effect of SBS modified Bitumen. In: International science index, vol 7, No 2

23. Hofko B, Alavi M, Grothe H, Jones D, Harvey J (2017) Repeatability and sensitivity of FTIR ATR spectral analysis methods for bituminous binders. Mater Struct 50(3):187. https://doi.org/10.1617/s11527-017-1059-x

24. Hofko B, Porot L, Falchetto Cannone A, Poulikakos L, Huber L, Lu X et al (2018) FTIR spectral analysis of bituminous binders: reproducibility and impact of ageing temperature. Mater Struct 51(2):45. https://doi.org/10.1617/ s11527-018-1170-X

25. Lu X, Isacsson U (2000) Artificial aging of polymer modified bitumens. J Appl Polym Sci 76(12):1811-1824

26. Xu J, Zhang A, Zhou T, Cao X, Xie Z (2007) A study on thermal oxidation mechanism of styrene-butadiene-styrene block copolymer (SBS). Polym Degrad Stab 92(9):1682-1691

27. Mills-Beale J, You Z, Fini E, Zada B, Lee C, Yap Y (2014) Aging influence on rheology properties of petroleum-based asphalt modified with biobinder. J Mater Civ Eng 26(2):358-366

28. Negulescu I, Muhammad L, Daly W, Abadie C, Cuero R, Daranga C, Glover I (2006) Chemical and rheological characterization of wet and dry aging of SBS copolymer 
modified asphalt cement: laboratory and field evaluation. J Assoc Asphalt Paving Technol 75:267-296

29. Cortizo M, Larsen D, Bianchetto H, Alessandrini J (2004) Effect of the thermal degradation of SBS copolymers during the ageing of modified asphalts. Polym Degrad Stab 86(2):275-282

30. Eberhardsteiner L, Füssl J, Hofko B, Handle F, Hospodka M, Blab R, Grothe H (2015) Towards a microstructural model of bitumen ageing behaviour. Int $\mathrm{J}$ Pavement Eng 16(10):939-949

31. Kumbargeri Y, Biligiri K (2015) A novel approach to understanding asphalt binder aging behavior using asphaltene proportion as a performance indicator. J Test Eval 44(1):20140490

32. Kumbargeri Y, Biligiri K (2016) Rational performance indicators to evaluate asphalt materials' aging characteristics. J Mater Civ Eng 28(12):04016157

33. Rek V, Vranjeś N, Barjaktaroví Z (2005) Evaluation of ageing properties of polymer modified bitumen. Mater Res Innovations 9(4):670-691

34. Handle F, Füssl J, Neudl S, Grossegger D, Eberhardsteiner L, Hofko B et al (2014) The bitumen microstructure: a fluorescent approach. Mater Struct 49(1-2):167-180. https://doi.org/10.1617/s11527-014-0484-3

35. Das PK, Kringos N, Birgisson B (2014) Microscale investigation of thin film surface ageing of bitumen. J Microsc 254(2):95-107

36. Airey GD, Brown SF (1998) Rheological performance of aged polymer modified bitumens. J Assoc Asph Pavement Technol 67:66-100

37. Ruan Y (2003) The effect of long-term oxidation on the rheological properties of polymer modified asphalts. Fuel 82(14):1763-1773

38. Ruan Y, Davison R, Glover C (2003) Oxidation and viscosity hardening of polymer-modified asphalts. Energy Fuels 17(4):991-998

39. Yao H, You Z, Li L, Goh S, Lee C, Yap Y, Shi X (2013) Rheological properties and chemical analysis of nanoclay and carbon microfiber modified asphalt with Fourier transform infrared spectroscopy. Constr Build Mater 38:327-337

40. Petersen JC (1984) Chemical composition of asphalt as related to asphalt durability: state of the art. Transp Res Rec J Transp Res Board 999:13-30

41. Petersen J, Glaser R (2011) Asphalt oxidation mechanisms and the role of oxidation products on age hardening revisited. Road Mater Pavement Des 12(4):795-819

42. Petersen JC (2009) A review of the fundamentals of asphalt oxidation. Transportation Research Board Circular E-C140
43. Page GC, Murphy KH, Ruth BE, Roque R (1985) Asphalt binder hardening-causes and effects. J Assoc Asph Paving Technol 140-167

44. Sisko AW, Brunstrum LC (1968) The rheological properties of asphalts in relation to durability and pavement performance. J Assoc Asph Paving Technol 37:28-39

45. Liu M, Ferry MA, Davison RR, Glover CJ, Bullin JA (1998) Oxygen uptake as correlated to carbonyl growth in aged asphalts and asphalt corbett fractions. Ind Eng Chem Res 37:4669-4674

46. Goodrich JL (1988) Asphalt and polymer modified asphalt properties related to the performance of asphalt concrete mixes (with discussion). J Assoc Asph Paving Technol 57:116-175

47. Oliver J, Tredera P (1997) The change in properties of polymer modified binders with simulated field exposure. J Assoc Asphalt Paving Technol 66:570-602

48. Ghavibazoo A, Abdelrahman M (2014) Effect of crumb rubber dissolution on low-temperature performance and aging of asphalt-rubber binder. Transp Res Rec J Transp Res Board 2445:47-55

49. Sugano M et al (2009) Thermal degradation mechanism of polymer modified asphalt. Chemical Engineering Transactions, vol 18

50. Lu X, Soenen H, Heyrman S, Redelius P (2013) Durability of polymer modified binders in porous asphalt pavements. In: Proceedings of the XXVIII international Baltic road conference

51. Singh B, Saboo N, Kumar P (2017) Effect of short-term aging on creep and recovery response of asphalt binders. J Transp Eng Part B Pavements 143(4):04017017

52. Hintz C, Velasquez RA, Li Z, Bahia HU (2011) Effect of oxidative aging on binder fatigue performance. J Assoc Asphalt Paving Technol 80:527-548

53. Lu X, Isacsson U (1999) Chemical and rheological characteristics of styrene-butadiene-styrene polymer-modified bitumens. Transp Res Rec J Transp Res Board 1661:83-92

54. Golalipour A (2011) Modification of multiple stress creep recovery test procedure usage in specification. Master Thesis, University of Wisconsin at Madison

55. Clopotel C, Bahia HU (2012) Importance of elastic recovery in the DSR for binders and mastics. Eng J 16(4):99-106

56. Anderson DA, Bonaquist R (2012) National highway research programme (NCHRP) report 709-investigation of short-term laboratory aging of neat and modified asphalt binders 